\title{
Effect of Combined Loading Due to Bending and Internal Pressure on Pipe Flaw Evaluation Criteria"
}

\author{
Naoki MIURA** $^{*}$ and Shinsuke SAKAI ${ }^{* * *}$ \\ ${ }^{* *}$ Central Research Institute of Electric Power Industry, \\ 2-11-1 Iwado-Kita, Komae-shi, Tokyo, 201-8511, Japan \\ E-mail: miura@criepi.denken.or.jp \\ ***University of Tokyo, \\ 7-3-1 Hongo, Bunkyo-ku, Tokyo, 113-8656, Japan \\ E-mail: sakai@fml.t.u-tokyo.ac.jp
}

\begin{abstract}
Considering a rule for the rationalization of maintenance of Light Water Reactor piping, reliable flaw evaluation criteria are essential for determining how a detected flaw will be detrimental to continuous plant operation. Ductile fracture is one of the dominant failure modes that must be considered for carbon steel piping and can be analyzed by elastic-plastic fracture mechanics. Some analytical efforts have provided various flaw evaluation criteria using load correction factors, such as the $Z$-factors in the JSME codes on fitness-for-service for nuclear power plants and the section XI of the ASME boiler and pressure vessel code. The present Z-factors were conventionally determined, taking conservativity and simplicity into account; however, the effect of internal pressure, which is an important factor under actual plant conditions, was not adequately considered. Recently, a J-estimation scheme, LBB.ENGC for the ductile fracture analysis of circumferentially through-wall-cracked pipes subjected to combined loading was developed for more accurate prediction under more realistic conditions. This method explicitly incorporates the contributions of both bending and tension due to internal pressure by means of a scheme that is compatible with an arbitrary combined-loading history. In this study, the effect of internal pressure on the flaw evaluation criteria was investigated using the new J-estimation scheme. The Z-factor obtained in this study was compared with the presently used Z-factors, and the predictability of the current flaw evaluation criteria was quantitatively evaluated in consideration of the internal pressure.
\end{abstract}

Key words: Fracture Criterion, Ductile Fracture, J-Integral, Fracture Mechanics, Pipe Flaw Evaluation, Fitness-For-Service, Z-Factor

\section{Introduction}

As the number of aged nuclear power plants is increasing, the substantiation of reliability as well as the assessment of the possible extensions of plant life is becoming important in Japan. Thus, the importance of appropriate and rationalized management of the plant life has been widely recognized. The Japan Society of Mechanical Engineers (JSME) has established a code for nuclear power generation facilities ${ }^{(1)}$, which provides the rules on the fitness-for-service for operating nuclear power plants. The concept of flaw evaluation forms the basis of the fitness-for-service rules, in which the crack propagation Eng., Vol.72, No.721, A (2006), pp.1389-1396 (Received 4 Apr., 2006) [DOl: 10.1299/jmmp.2.685] 
from detected flaws up to the end of the plant life or the next inspection period is predicted to determine the necessity of repairing or replacing the components of concern. Focusing on the piping typical of component elements, the flaw evaluation addresses acceptance criteria to predict the load and mode at the final failure status by the use of elastic-plastic fracture analysis.

Since piping is essentially used for the transportation of fluid, it is subjected to tensile load due to internal pressure under normal operating conditions. Considering seismic force as an external load, piping is additionally subjected to a bending load as a critical loading mode. Therefore, combined loading consisting of tension and bending is the basic loading condition to be considered for pipe failure. However, pipe fracture behavior under combined loading has not been adequately examined. The effect of combined loading is not addressed in the present flaw evaluation criteria, but is treated in a conventional manner. The authors recently developed a J-estimation scheme, LBB.ENGC, for the ductile fracture analysis of circumferentially through-wall-cracked pipes subjected to combined loading ${ }^{(2)-(4)}$. This method incorporates the contributions of both tension and bending using explicit expressions. The effectivity of the method was validated through a comparison with the results of large-scale fracture experiments on cracked pipes under a combined loading condition ${ }^{(4)}$.

In the present study, the effect of combined loading on the flaw evaluation criteria for a common carbon steel piping material was quantitatively investigated using LBB.ENGC. More accurate flaw evaluation criteria taking combined loading into account were derived, and the possible rationalization of maintenance by using new methods was investigated by a comparison with the flaw evaluation criteria in the presently used codes.

\section{Outline of Ductile Fracture Evaluation}

Figure 1 shows the schematic of a cracked pipe of concern. $R$ is the mean radius, $t$ is the pipe thickness, and $2 \theta$ is the total crack angle. The circumferentially through-wall-cracked pipe is subjected to bending moment $M$ and tensile load $T$ due to internal pressure, and these loadings are represented by bending stress $\sigma_{b}\left(=M /\left(\pi R^{2} t\right)\right)$ and tensile stress $\sigma_{t}(=T /(2 \pi R t))$, respectively. The parameter $s$ is defined as $\sigma_{t}$ normalized by the material flow stress $\sigma_{f}$ and is used for simplicity hereinafter.

The rotation $\phi$, due to the crack may be written as the sum of its elastic and plastic components $\phi_{E L}$ and $\phi_{P L}$ :

$$
\phi=\phi_{E L}+\phi_{P L}
$$

$\phi_{E L}$ is related to the strain energy using Castigliano's theorem, and is expressed as

$$
\begin{aligned}
& \phi_{E L}=\frac{\sigma_{b}}{E} I_{b}(\theta)+\frac{\sigma_{t}}{E} I_{t}(\theta) \\
& I_{b}(\theta)=4 \int_{0}^{\theta} \theta F_{b}^{2}(\theta) d \theta \\
& I_{t}(\theta)=4 \int_{0}^{\theta} \theta F_{b}(\theta) F_{t}(\theta) d \theta .
\end{aligned}
$$

where $E$ is Young's modulus, and $F_{b}(\theta)$ and $F_{t}(\theta)$ are the compliance functions of stress

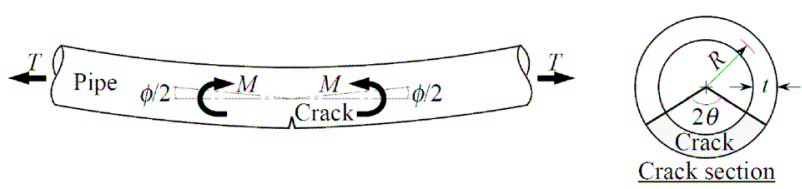

Fig. 1 Circumferentially through-wall-cracked pipe subjected to combined tension and bending 
intensity factors $K_{b}$ and $K_{t}$ for bending and tension, respectively, which are defined as functions of $\theta$ by

$$
F_{b}(\theta)=\frac{K_{b}}{\sigma_{b} \sqrt{\pi R \theta}}, \quad F_{t}(\theta)=\frac{K_{t}}{\sigma_{t} \sqrt{\pi R \theta}} .
$$

The solutions of $K_{b}$ and $K_{t}$ are obtained by Klecker et al. ${ }^{(5)}$ as an example.

For the evaluation of $\phi_{E L}$, beam theory can be applied to a pipe of nonlinear elastic material on the basis of the deformation theory of plasticity. The relation between stress $\sigma$ and strain $\varepsilon$ is defined by the following power law:

$$
\varepsilon / \varepsilon_{0}=\alpha\left(\sigma / \sigma_{0}\right)^{n}, \quad \varepsilon_{0}=\sigma_{0} / E
$$

Furthermore, assuming that the existence of a crack can be replaced with a pipe section with reduced thickness $t_{e}$ and that the pipe section can be used to simulate the reduction of the compliance due to the existence of the crack, the following evaluation equations are derived $^{(4)}$ :

$$
\begin{aligned}
& \phi_{P L}=\left(\frac{t}{t_{e}}\right)^{n-1}\left(\frac{\pi}{4 \hat{K}_{M}}\right)^{n} \alpha \frac{\kappa^{n}}{\kappa(n=1)}\left(\frac{\sigma_{b}}{\sigma_{0}}\right)^{n-1} \phi_{E L} \\
& {\left[1-\left(1-\frac{\pi}{4}\right) \sin 2 \theta\right] \frac{\cos \left(\frac{\pi}{2} s\right)}{\cos \frac{\theta}{2}-\frac{1}{2} \sin \theta}, \quad \text { for } \theta<\frac{\pi}{4}} \\
& \frac{t}{t_{e}}=\left\{\begin{array}{l}
\frac{\pi}{4} \frac{\cos \left(\frac{\pi}{2} s\right)}{\cos \frac{\theta}{2}-\frac{1}{2} \sin \theta}, \quad \text { for } \theta \geq \frac{\pi}{4} \\
\kappa=\frac{1}{2}\left\{1+\left[1+\frac{16 \hat{K}_{M}\left(\hat{K}_{T} g_{T}-\hat{K}_{M} g_{M}\right)\left(\sigma_{t} / \sigma_{b}\right)^{2}}{\hat{K}_{T}^{2} g_{T}^{2}}\right]^{1 / 2}\right.
\end{array}\right\} \\
& \hat{K}_{M}=\frac{\left[\sqrt{\pi} \frac{\Gamma\left(\frac{1}{2 n}+1\right)}{2}, \hat{K}_{T}=\frac{\sqrt{\pi}}{2} \frac{\Gamma\left(\frac{1}{2 n}+\frac{3}{2}\right)}{\Gamma\left(\frac{1}{2 n}+1\right)}\right.}{g_{M}=\frac{n+1}{n} \frac{2}{\pi}, \frac{g_{T}}{\pi} \frac{1}{n}+\frac{2}{\pi} .}
\end{aligned}
$$

where $\Gamma$ is the Gamma function.

The J-integral, $J$, is also divided into its elastic and plastic components $J_{E L}$ and $J_{P L}$.

$$
J=J_{E L}+J_{P L}
$$

$J_{E L}$ is directly obtained from the stress intensity factor $K_{I}$ under a plane stress condition:

$$
J_{E L}=K_{I}^{2} / E .
$$

On the basis of the fact that $J$ is equivalent to the release rate of potential energy for a nonlinear elastic material and the assumption that the plastic deformation is a function of 
only the net-section stress, $J_{P L}$ can be calculated by the following equations ${ }^{(2)}$ :

$$
\begin{aligned}
& J_{P L}=\int_{0}^{\phi_{P L}} \eta_{p} M d \phi_{P L}+2 \int_{\theta_{0}}^{\theta} \gamma_{p} J_{P L} d \theta+2 \int_{s_{0}}^{s} \mu_{p} J_{P L} d s \\
& \eta_{p}=-\frac{\partial h_{p} / \partial \theta}{2 R t h_{p}} \\
& \gamma_{p}=\frac{\partial^{2} h_{p} / \partial \theta^{2}}{2 \partial h_{p} / \partial \theta} \\
& \mu_{p}=\frac{\partial^{2} h_{p} / \partial s \partial \theta}{2 \partial h_{p} / \partial \theta} \\
& h_{p}=\cos \left(\frac{\theta}{2}+\frac{\pi}{2} s\right)-\frac{1}{2} \sin \theta .
\end{aligned}
$$

The second term on the right side of Eq. (14) corresponds to the contribution of the crack growth to $J_{P L}$ and is 0 for a stationary crack. The third term on the right side of the equation corresponds to the contribution of the tensile load variation to $J_{P L}$ and is 0 under constant tensile loading.

By integrating the load-rotation relation given by Eqs. (1), (2), and (7), the J-integral provided by Eqs. (12) to (14), and the J-resistance curve,

$$
J=J_{R}(\Delta a)
$$

which is introduced as an additional criterion of ductile crack extension, then the loads, rotation, J-integral, and crack extension are uniquely related to each other, which means that the prediction of fracture behavior in consideration of both combined loading and crack extension can be achieved.

\section{Derivation of Pipe Flaw Evaluation Criteria}

\subsection{Basic Concept}

Figure 2 shows a flowchart of pipe flaw evaluation. In the first step of evaluation, characterized flaws detected by nondestructive tests are compared with the allowable flaw sizes in the acceptance standards. If the characterized flaws are smaller than the allowable flaw sizes, they are acceptable for continued service. Otherwise, the flaws must be repaired or evaluated by the second step of evaluation. In this step, the extent of crack

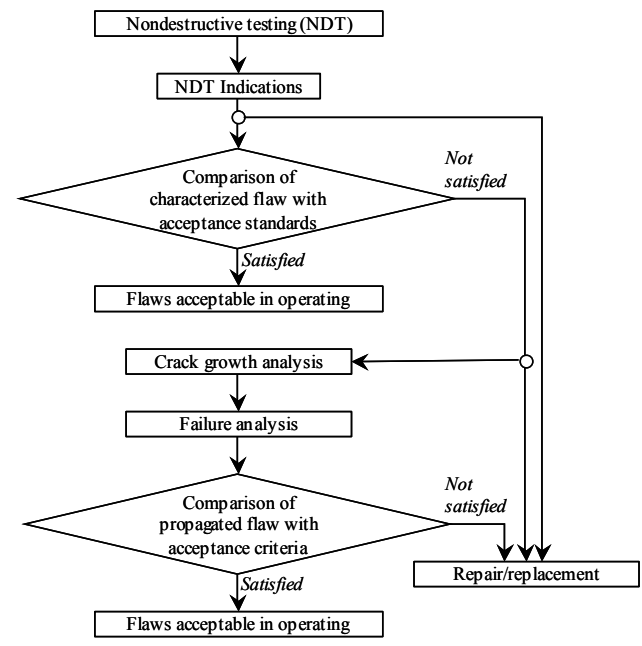

Fig. 2 Flowchart of pipe flaw evaluation 
growth up to the next inspection or the end of the service lifetime is determined, and failure analysis is subsequently conducted. The predicted propagated flaws are compared with the critical flaw sizes in the acceptance criteria. If they are smaller than the critical flaw sizes, they are acceptable for continued service during the evaluated time period. Otherwise the flaws must be removed or repaired.

In the above evaluation procedure, critical flaw sizes in the acceptance criteria must be determined in accordance with the expected failure mode under the specific conditions. When a pipe material exhibits a relatively high J-resistance, final failure is due by plastic collapse $^{(6)}$. The failure load is then identical to the plastic collapse load, which can be predicted by limit load analysis based on the net-section collapse criterion. The net-section collapse load is obtained from the equilibrium condition of the admissible stress distribution on the cracked section. Thus, it can be easily evaluated once the lower limit of the flow stress is identified.

In contrast, the J-resistance is often lower than the driving force of crack growth for carbon steel piping, and the failure occurs after a certain amount of stable crack growth. In such a case, the failure load is evaluated by elastic-plastic fracture analysis using the J-estimation scheme.

\subsection{Significance of Z-factor and Consideration of Combined Loading}

In the present flaw evaluation criteria, the $Z$-factor $(Z)$ is used to determine the critical flaw sizes for ductile fracture. $Z$ is defined as the ratio of the net-section collapse load to the failure load. It is equal to unity for plastic collapse and larger than unity for ductile fracture. $Z$ indicates the degree of the reduction of the load-carrying capacity from the net-section collapse load. If $Z$ is previously identified, the failure load for ductile fracture can be easily evaluated as the net-section collapse load divided by $Z$. Alternatively, a critical crack size for a certain load under the plastic collapse condition can be directly converted into the same critical crack size for the load multiplied by $Z$ under the ductile fracture condition.

The concept of the Z-factor was first introduced in the Section XI of the ASME Boiler and Pressure Vessel Code ${ }^{(7)}$. $Z$ was formulated as a function of the nominal diameter $O D$ (in inches) and the radius-to-thickness ratio $R / t^{(6)(8)}$. The Z-factor in the ASME Code, $Z_{A S M E}$, for a ferritic steel base metal pipe is

$$
\begin{aligned}
& Z_{A S M E}=1.20[1+0.021 A(O D-4)] \\
& A=\left\{\begin{array}{l}
{[0.125(R / t)-0.25]^{0.25}, \quad \text { for } 5 \leq R / t \leq 10} \\
{[0.4(R / t)-3.0]^{0.25}, \quad \text { for } 10<R / t \leq 20}
\end{array}\right. \text {. }
\end{aligned}
$$

The above equations are applicable when $O D$ is between 4 and 37 inches. Another $Z$-factor is given in the JSME Code ${ }^{(1)}$ taking the properties of materials used in Japan into account. The Z-factor in the JSME Code, $Z_{J S M E}$, for a ferritic steel base metal pipe is

$$
Z_{J S M E}=0.2885 \log _{10} O D+0.9573
$$

The above equations are applicable when 6 inches $\leq O D \leq 30$ inches. The applicable range of $R / t$ is not obvious; however, it is approximately between 4 and 9 . Note that both Eqs. (20) and (22) consider pure bending load only, and the effect of combined loading is not considered.

It is expected that a new Z-factor taking the effect of combined loading into account can be derived using LBB.ENGC. The Z-factor under combined loading $Z_{C}$ is defined as 


$$
Z_{C}=\operatorname{Max}\left[1, M_{N S C} / M_{\max }\right]
$$

where $M_{N S C}$ is the net-section collapse moment given by

$$
M_{N S C}=4 \sigma_{f} R^{2} t h_{p}(\theta, s)
$$

and $h_{p}$ is given in Eq. (18). $M_{\max }$ in Eq. (23) is the maximum moment predicted using LBB.ENGC.

\section{Analysis of Z-factor}

\subsection{Conditions of Analysis}

LBB.ENGC was applied to analyze the Z-factor under combined loading, $Z_{C}$. The material properties used for the analysis were determined on the basis of the Japanese carbon steel pipe database ${ }^{(9)(10)}$. The stress-strain relation for STS480 carbon steel at $300^{\circ} \mathrm{C}$, and the J-R curve for STS 410 carbon steel at $300^{\circ} \mathrm{C}$ were selected to give the most conservative values of $Z_{C}$. Table 1 shows the material properties used for the analysis.

$\theta_{0}, O D$, the nominal thickness (or the radius-to-thickness ratio $R / t$ ), and the normalized membrane stress $P_{m} / S_{m}$ (where $P_{m}$ is the membrane stress due to internal pressure and $S_{m}$ is the design stress intensity) were considered as the parameters affecting $Z_{C}$. The range of $\theta_{0}$ was 5 to $90^{\circ}$, and the range of $O D$ was 2.5 to 30 inches. The nominal thickness is fixed to Sch 80 but is sometimes increased to Sch $160 . P_{m} / S_{m}$ was set to 0 (no internal pressure), 0.34 (maximum value obtained from actual plant surveys ${ }^{(9)(10)}$ ), and 0.5 (maximum allowable value under normal operating conditions). A finer subdivision was also applied as necessary to observe the effect of $P_{m} / S_{m}$ on $Z_{C}$.

\subsection{Results of Analysis}

Figures 3(a) and (b) show typical predicted relations between moment and rotation for 6- and 26-inch-diameter pipes, respectively. These relations have a similar configuration, and the maximum moment decreases with increasing $P_{m} / S_{m}$.

Figures 4(a) to (c) show the effect of $O D$ on $Z_{C}$ for the three values of $P_{m} / S_{m}$. As defined in Eq. (23), $Z_{C}$ is effective only in the case of $Z_{C}>1$; however, the ratio of the net-section collapse moment to the predicted maximum moment obtained from LBB.ENGC is regarded here as the conventional Z-factor. $\quad \theta_{0}$ was fixed at $15,30, \cdots, 90^{\circ}$ and $O D$ was

\begin{tabular}{|c|c|c|c|c|c|c|c|c|}
\hline \multirow{2}{*}{$\begin{array}{l}\text { Young's } \\
\text { modulus }\end{array}$} & \multirow{2}{*}{$\begin{array}{l}\text { Flow } \\
\text { stress }\end{array}$} & \multicolumn{3}{|c|}{ Stress-strain relation ${ }^{*}$} & \multicolumn{3}{|c|}{$\mathrm{J}-\mathrm{R}$ curve ${ }^{* *}$} & \multirow{2}{*}{$\begin{array}{c}\text { Design } \\
\text { stress } \\
\text { intensity }\end{array}$} \\
\hline & & $\sigma_{0}$ & $\alpha$ & $n$ & $J_{\text {in }}$ & $C$ & $m$ & \\
\hline $196 \mathrm{GPa}$ & $389 \mathrm{MPa}$ & $244 \mathrm{MPa}$ & 4.412 & 3.389 & $0.119 \mathrm{MN} / \mathrm{m}$ & 136.3 & 0.9234 & $139 \mathrm{MPa}$ \\
\hline
\end{tabular}

Table 1 Material properties used for analysis

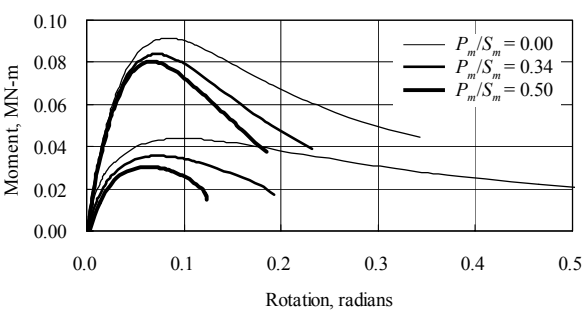

(a) 6-inch-diameter, Sch 80 pipes

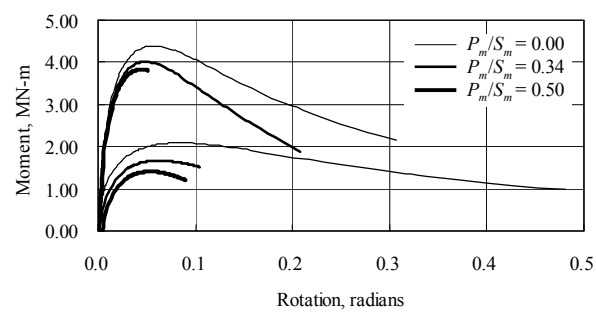

(b) 26-inch-diameter, Sch 80 pipes

Fig. 3 Examples of predicted relations between moment and rotation 
varied from 2.5 to 30 -inches. The case when $P_{m} / S_{m}=0.50$ and $\theta_{0}=90^{\circ}$ was excluded because these values result in plastic collapse due to pure tension. The nominal thickness was Sch 80 . These figures show that a larger $O D$ generally gives a larger $Z_{C}$ regardless of the value of $P_{m} / S_{m}$ or $\theta_{0}$. The dependence of $O D$ on $Z_{C}$ seems to be more significant when $\theta_{0}$ is larger. $O D$, as well as $P_{m} / S_{m}$ and $\theta_{0}$, determines whether or not $Z_{C}$ is larger than unity. The change in the slope at a diameter of 30 -inches is caused by the larger value of $R / t$ for this diameter.

Figures 5(a) to (c) show the effect of $\theta_{0}$ on $Z_{C}$ for the three values of $P_{m} / S_{m}$. OD was fixed at $2.5,6,16$, and 26 inches and $\theta_{0}$ was varied from 5 to $90^{\circ}$. The case when $P_{m} / S_{m}=$ 0.50 and $\theta_{0}=90^{\circ}$ was also excluded. The nominal thickness was Sch 80 . These figures show that $Z_{C}$ increases monotonically with increasing $\theta_{0}$ when $P_{m} / S_{m}=0.00$, however, $Z_{C}$ attains its maximum value at a certain value of $\theta_{0}$ in the case of positive $P_{m} / S_{m}$. This value of $\theta_{0}$ ranges between 55 to $70^{\circ}$ and depends on both $P_{m} / S_{m}$ and $O D$.

Figure 6 shows the effect of $R / t$ on $Z_{C}$ for the three different values of $P_{m} / S_{m}$. OD and $\theta_{0}$ were fixed at 26 inches and $60^{\circ}$, respectively. The nominal thickness was varied from Sch 80 to 160 . $R / t$ was 9.2 for Sch 80 and 4.6 for Sch 160 . It can be seen from the figure that $Z_{C}$ increases almost linearly with increasing $R / t$.

Figure 7 shows the effect of $P_{m} / S_{m}$ on $Z_{C}$. $O D$ was fixed at 6,16 , and 26 inches and $\theta_{0}$ was fixed at $60^{\circ}$. $\quad P_{m} / S_{m}$ was varied from 0.00 to 0.50 . The figure also shows that $Z_{C}$ has

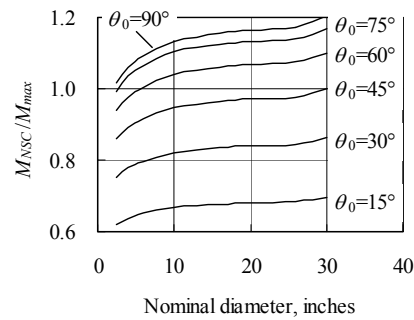

(a) $P_{m} / S_{m}=0.00$

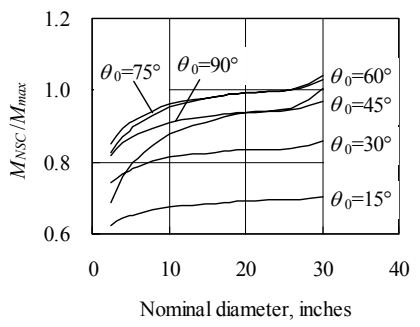

(b) $P_{m} / S_{m}=0.34$

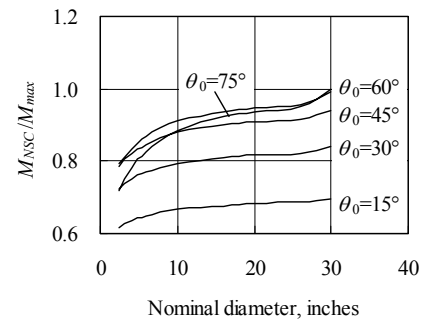

(c) $P_{m} / S_{m}=0.50$

Fig. 4 Effect of nominal diameter on Z-factor

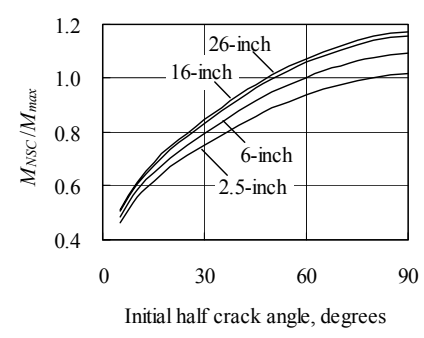

(a) $P_{m} / S_{m}=0.00$

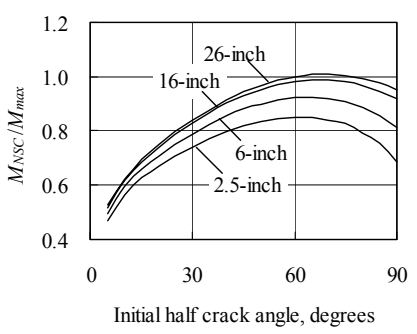

(b) $P_{m} / S_{m}=0.34$

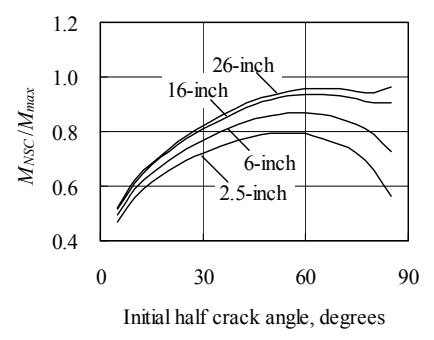

(c) $P_{m} / S_{m}=0.50$

Fig. 5 Effect of initial half crack angle on Z-factor

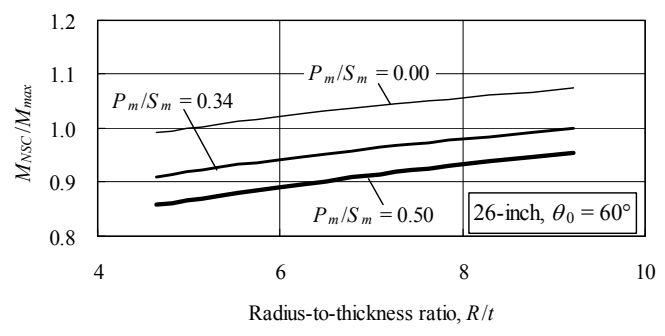

Fig. 6 Effect of $R / t$ on Z-factor

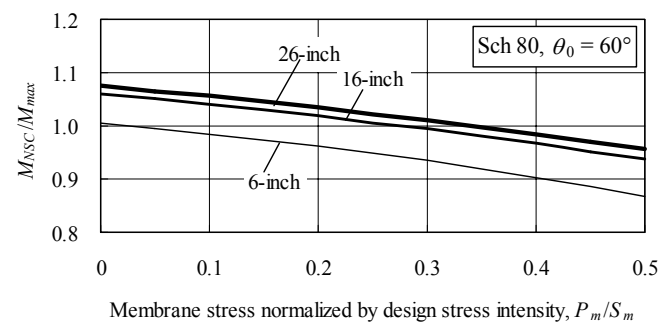

Fig. 7 Effect of $P_{m} / S_{m}$ on Z-factor 
a linear relationship with $P_{m} / S_{m}$. It can be ascertained that a similar relationship is also satisfied for different $O D, \theta_{0}$, and $R / t$ values. In previous studies ${ }^{(9)(10)}$, an increase in $P_{m} / S_{m}$ due to internal pressure was shown to slightly reduce the load-correction factor. This is because the R6 procedure option $2^{(11)}$ was applied to conveniently identify the Z-factor under combined loading, although the accuracy of evaluation using this method has not been adequately confirmed. Therefore, the R6 procedure may give a too conservative prediction under a combined loading condition.

\subsection{Formulation of Z-Factor}

On the basis of the results of the analysis, an approximate expression for $Z_{C}$ under combined loading was formulated. $Z_{C}$ can be generally expressed as a function of $O D, \theta_{0}$, $R / t$, and $P_{m} / S_{m}$ :

$$
Z_{C}=Z_{C}\left(O D, \theta_{0}, R / t, P_{m} / S_{m}\right)
$$

However, it would not be appropriate to consider all the parameters in Eq. (25) from the viewpoint of codification. In this study, the contributions of the parameters to $Z_{C}$ are addressed as follows. The dominant parameter affecting $Z_{C}$ is assumed to be $O D$. Since the effect of $\theta_{0}$ is difficult to incorporate in the expression in a consistent manner, $Z_{C}$ is conservatively replaced with its maximum value for arbitrary values of $\theta_{0}$. The effects of both $R / t$ and $P_{m} / S_{m}$ seem to be independent of other factors according to Figs. 6 and 7 , respectively. Consequently, Eq. (25) can be rewritten as

$$
Z_{C}=f_{1}\left(P_{m} / S_{m}\right) f_{2}(R / t) f_{3}(O D) \text {. }
$$

In the above equation, functions $f_{1}$ and $f_{2}$ were obtained by the linear approximations by the least-squares method using the result of the analysis, and function $f_{3}$ was determined by assuring a linear relation of $Z_{C}$ with $\log _{10}(O D)$ excluding the contributions of both $P_{m} / S_{m}$ and $R / t$. Thus, the formula used to evaluate $Z_{C}$ under combined loading is given by the following equation:

$$
Z_{C}=\left[1.00-0.226 \frac{P_{m}}{S_{m}}\right]\left[0.826+0.0189 \frac{R}{t}\right]\left[0.977+0.0707 \log _{10} O D\right]
$$

Again note that $Z_{C}$ should be unity in the case of $Z_{C}<1$. The applicable range of this equation is limited to the range of the parameters in the analysis, that is, 2.5 to 30 inches for $O D$, Sch 80 to 160 for nominal thickness, $\theta_{0} \leq 60^{\circ}$, and $P_{m} / S_{m} \leq 0.5$.

Figure 8 shows a comparison of the values of $Z_{C}$ predicted using Eq. (27) and the calculated values for each analysis condition. Results when $Z_{C}<1$ are also plotted for comparison. The predicted values of $Z_{C}$ are in good agreement with the calculated values for a wide range of $O D$ and $P_{m} / S_{m}$, and the difference is less than $3 \%$.

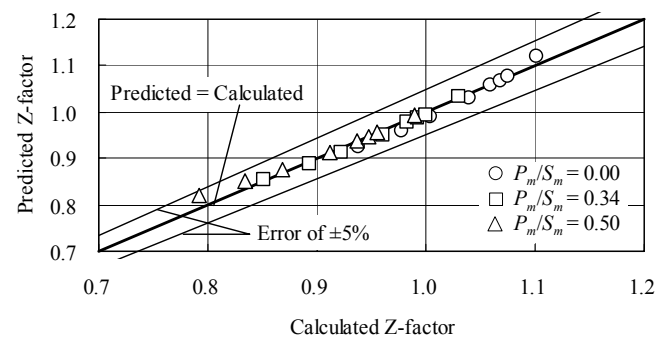

Fig. 8 Comparison between calculated and predicted Z-factors 


\subsection{Comparison with Present Z-Factors}

The proposed formula for $Z_{C}$ under combined loading in Eq. (27) was compared with the present $Z$-factors, $Z_{A S M E}$ in Eq. (20) and $Z_{J S M E}$ in Eq. (22), as shown in Figs. 9(a) and (b). Results for $Z_{C}<1$ are also plotted. When $R / t=5$ and 10 , the values of $Z_{C}$ are always less than the other Z-factors independently of $O D$ or $P_{m} / S_{m}$. Since the material properties used to develop $Z_{J S M E}$ and $Z_{C}$ are essentially the same, the difference between $Z_{J S M E}$ and $Z_{C}$ when $P_{m} / S_{m}=0$ results from the accuracy and conservativity of the evaluation methods. In other words, the R6 procedure option $2^{(11)}$ used to develop $Z_{J S M E}$ gives conservative predictions of failure loads, while LBB.ENGC, used to develop $Z_{C}$, may predict more accurate failure loads.

As a result, it was ascertained that the presently used Z-factors, which do not take combined loading into consideration, were still conservative under a combined loading condition, and the new Z-factor under combined loading promises more reliable flaw evaluation.

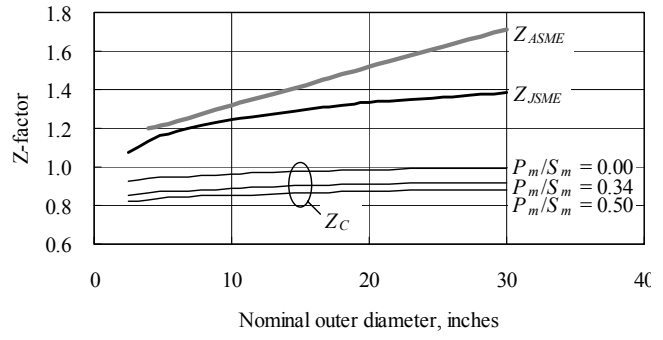

(a) $R / t=5$

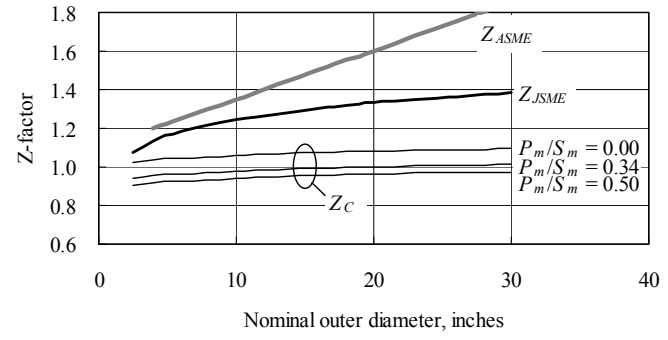

(b) $R / t=10$

Fig. 9 Comparison between proposed and present Z-factors

\section{Conclusions}

In this study, a Z-factor for carbon steel pipes taking combined loading into account was investigated. The J-estimation scheme, LBB.ENGC, developed to evaluate the ductile fracture behavior of circumferentially through-wall-cracked pipes subjected to combined loading, was used to analyze the Z-factor. The effect of internal pressure as well as other factors on the Z-factor was quantitatively examined. It was found that the Z-factor was generally larger for a larger diameter, larger crack angle, smaller thickness, and lower pressure. A formula to evaluate the Z-factor under combined loading was developed on the basis of the results of the analysis, and was compared with the present Z-factors. As a result, it was ascertained that the presently used Z-factors, which do not take combined loading into consideration, were still conservative under a combined loading condition. The new Z-factor under combined loading promises more reliable flaw evaluation.

The authors would like to express their appreciation to Dr. Satoshi Izumi for valuable comments.

\section{References}

(1) The Japan Society of Mechanical Engineers, Codes for Nuclear Power Generation Facilities -Rules of Fitness-for-Service for Nuclear Power Plants-, JSME S NA1-2004 (2004).

(2) Miura, N. and Wilkowski, G., J-R Curves from Circumferentially Through-Wall-Cracked Pipe Tests Subjected to Combined Bending and Tension -Part I: Theory and Numerical Simulation, Transactions of the ASME, Journal of Pressure Vessel Technology, Vol. 120 (1998), pp. 406-411. 
(3) Miura, N. and Wilkowski, G., J-R Curves from Circumferentially Through-Wall-Cracked Pipe Tests Subjected to Combined Bending and Tension -Part II: Experimental and Analytical Validation, Transactions of the ASME, Journal of Pressure Vessel Technology, Vol. 120 (1998), pp. 412-417.

(4) Miura, N., Approximate Evaluation Method for Ductile Fracture Analysis of Circumferentially Through-Wall-Cracked Pipe Subjected to Combined Bending and Tension, Nuclear Engineering and Design, Vol. 191 (1999), pp. 177-194.

(5) Klecker, R. et al., NRC Leak-Before-Break (LBB.NRC) Analysis Method for Circumferentially Through-Wall Cracked Pipes Under Axial Plus Bending Loads, NUREG/CR-4572 (1986).

(6) Novetech Corporation, Evaluation of Flaws in Ferritic Piping, EPRI NP-6045, Final Report (1988).

(7) ASME Boiler and Pressure Vessel Code: Section XI, Rules for Inservice Inspection of Nuclear Power Plant Components (2004).

(8) Section XI Task Group for Piping Flaw Evaluation, Evaluation of Flaws in Austenitic Steel Piping, EPRI NP-4690-SR, Special Report (1986).

(9) Nuclear Power Engineering Test Center, Proving Test on the Integrity of Carbon Steel Piping in LWRs, Summary Report of Proving Tests on the Reliability for Nuclear Power Plant (1989).

(10) Asada, Y. et al., Leak-Before-Break Verification Test and Evaluations of Crack Growth and Fracture Criterion for Carbon Steel Piping, International Journal of Pressure Vessel and Piping, Vol. 43 (1990), pp. 379-397.

(11) Milne, I. et al., Assessment of the Integrity of Structures Containing Defects, CEGB R/H/R6 -Revision 3 (1987). 